\title{
Concavity of Utility, Concavity of Welfare, and Redistribution of Income
}

\section{Citation}

Louis Kaplow, Concavity of Utility, Concavity of Welfare, and Redistribution of Income, 17 Int'l Tax \& Pub. Fin. 25 (2010).

\section{Published Version}

http://link.springer.com/article/10.1007\%2Fs10797-008-9097-5\#page-1

\section{Permanent link}

http://nrs.harvard.edu/urn-3:HUL.InstRepos:12027853

\section{Terms of Use}

This article was downloaded from Harvard University's DASH repository, and is made available under the terms and conditions applicable to Open Access Policy Articles, as set forth at http:// nrs.harvard.edu/urn-3:HUL.InstRepos:dash.current.terms-of-use\#OAP

\section{Share Your Story}

The Harvard community has made this article openly available.

Please share how this access benefits you. Submit a story.

\section{Accessibility}




\title{
Harvard
}

John M. Olin Center for Law, Economics, and Business

\author{
CONCAVITY OF UTILITY, \\ CONCAVITY OF WELFARE, \\ AND REDISTRIBUTION OF INCOME \\ Louis Kaplow
}

Discussion Paper No. 437

$09 / 2003$

Harvard Law School

Cambridge, MA 02138

The Center for Law, Economics, and Business is supported by a grant from the John M. Olin Foundation.

This paper can be downloaded without charge from:

The Harvard John M. Olin Discussion Paper Series: http://www.law.harvard.edu/programs/olin_center/

The Social Science Research Network Electronic Paper Collection:

http://papers.ssrn.com/abstract_id $=463241$ 
JEL Classes D31, D63, H21, H23

\title{
Concavity of Utility, Concavity of Welfare, and Redistribution of Income
}

\author{
Louis Kaplow*
}

\begin{abstract}
The marginal social value of income redistribution is understood to depend on both the concavity of individuals' utility functions and the concavity of the social welfare function. In the pertinent literatures, notably on optimal income taxation and on normative inequality measurement, it seems to be accepted that the role of these two sources of concavity is symmetric with regard to the social concern about inequality in the distribution of income. Direct examination of the question, however, reveals that this is not the case. Concavity of utility has a simple, direct effect on the marginal social value of redistribution, as might be expected, whereas concavity of the social welfare function has a more subtle influence, one that in some cases may not be very significant. The implications of this difference are examined for some standard forms of utility and welfare functions, including particular versions that appear in the optimal income taxation literature.
\end{abstract}

\footnotetext{
*Harvard University and National Bureau of Economic Research. I am grateful to Steven Shavell for comments and the John M. Olin Center for Law, Economics, and Business at Harvard University for financial support.
} 


\title{
Concavity of Utility, Concavity of Welfare, and Redistribution of Income
}

\author{
Louis Kaplow \\ (C) 2003 Louis Kaplow. All rights reserved.
}

\section{Introduction}

The marginal social value of income redistribution is an important concept in applied welfare economics and plays a central role in literatures on optimal income taxation and normative inequality measurement. ${ }^{1}$ In colloquial terms, this value indicates how leaky a bucket society is willing to tolerate when deciding how much income redistribution is appropriate. Thus, if a dollar has twice the social value to the poorer recipient of a transfer than to the richer individual who funds it through taxation, further redistribution would increase social welfare if and only if less than half of what the rich person pays is wasted on account of distortion.

In the standard formulation, this marginal social value of income redistribution is derived from a social welfare function that is taken to be the sum or integral of $W(U)$, where welfare $W$ is a concave function of an individual's utility $U$ (notation referring to particular individuals is omitted throughout for ease of exposition). ${ }^{2}$ Likewise, the utility functions $U(y)$ are taken to depend on each individual's disposable income $y$ (and also on labor effort, such as in optimal income tax analysis). Accordingly, the marginal social value of income redistribution - the extent to which a dollar is deemed to be worth more to a poorer individual than to a richer one depends on the concavity of the functions $U$ and $W$.

The concavity of $U(y)$ indicates the rate at which individuals' marginal utility of income falls as income rises; a greater rate of diminution favors more redistribution, ceteris paribus. ${ }^{3}$ The degree of concavity of $U(y)$, it should be noted, is an empirical question because it is reflected, for example, in behavior under uncertainty; it is often measured by the coefficient of relative risk aversion (CRRA). ${ }^{4}$ The concavity of $W(U)$, by contrast, involves a value judgment that indicates society's aversion to inequality in the distribution of utilities. For example, a utilitarian social welfare function implies that $W$ is linear in $U$; a greater social preference for equality implies that $W$ is strictly concave in $U$. Standard functional forms for $W(U)$ are often analogous to those used for $U(y)$ - for example, reflecting constant relative aversion to inequality

\footnotetext{
${ }^{1}$ See, for example, Atkinson (1970, 1973), Atkinson and Stiglitz (1980), Blackorby, Bossert, and Donaldson (1999), Mirrlees (1971), Slemrod et al. (1984), Stern (1976), and Tuomala (1990).

${ }^{2}$ For example, one may have a social welfare function of the form $\int W(U(n)) f(n) d n$,

where $n$ reflects an individual's type (perhaps income-earning ability) and $f(n)$ is a density function.

${ }^{3}$ Other characteristics of individuals' utility functions may favor or oppose redistribution, such as certain forms of sympathy or envy. See, for example, Hochman and Rodgers (1969) and Boskin and Sheshinski (1978). These largely orthogonal complications are ignored here.

${ }^{4}$ For empirical literature measuring individuals' CRRA's, see, for example, Barsky et al. (1997), Campbell (1996), Choi and Menezes (1992), and Kocherlakota (1996).
} 
in the distribution of utilities - and thus similar measures of concavity, analogous to CRRA for utility functions, are employed.

In applying this framework, such as in optimal income tax simulations, it is common to employ some simple composite or reduced-form function $W(y)$, which allows one to determine directly the marginal social welfare contribution of a dollar to an individual with disposable income $y$. Different concavity parameters for this reduced-form function - typically, analogues to CRRA - are to be interpreted as corresponding to some combination of individual risk aversion (equivalently, rate of diminishing marginal utility of income) and social utilityinequality aversion. See, for example, Atkinson (1973), Atkinson and Stiglitz (1980) and Stern (1976). Thus, if a simulation uses a $W(y)$ with constant relative income-inequality aversion of 2 , the reader is invited to interpret the result as applicable to a utilitarian welfare function with individuals having constant CRRA of 2 , an egalitarian welfare function with an inequality aversion parameter of 2 with risk-neutral individuals, or any combination in between. In sum, it is implicitly assumed that the role of concavity of utility and concavity of welfare are symmetric, perhaps in some rough sense additive. ${ }^{5}$

This conventional understanding of the roles of concavity of utility and of welfare in determining the marginal social value of income redistribution is incomplete and potentially misleading. In section 2, the relationship between the concavity of the composite function $\mathrm{W}(U(y))$ or $W(y)$ and the separate welfare function $W(U)$ and utility function $U(y)$ is analyzed. A simple derivation reveals that the concavity of $U(y)$ contributes to the concavity of $W(y)$ in the straightforward manner that one would expect whereas the effect of the concavity of $W(U)$ on the concavity of $W(y)$ is more subtle (specifically, as long as individuals are not risk neutral, so that $U(y)$ is strictly concave $)^{6}$

The intuition behind this asymmetry can be explained as follows. Suppose that $U(y)$ is strictly concave in $y$; perhaps the functional form is $\ln y$. Suppose further that disposable income in the population is reasonably high. In this case, $U(y)$ is relatively flat in the relevant region. The concavity of $W(U)$ indicates the degree of aversion to inequality in utility levels, but since utility levels will be nearly equal, this concavity will not be very important. That is, introducing moderate degrees of concavity of $W(U)$ will not significantly increase the social preference for

\footnotetext{
${ }^{5}$ The question of the precise relationship of the concavity of $W(y)$ to that of $U(y)$ and $W(U)$ does not seem to have received direct attention. Some papers, such as Atkinson (1970, 1973), simply present the reduced-form function $W(y)$ without elaboration. Others, for example, Mirrlees (1971), Slemrod et al. (1984), and Stern (1976), explicitly state a separate $U(y)$ function and $W(U)$ function, yet as discussed in section 4 , the interpretation is not always clear, especially in light of the fact that the $U(y)$ functions often employed are quasi-linear (homogenous of degree one in consumption and leisure).

${ }^{6}$ This interpretation could be objected to because the manner in which the concavity of $W(U)$ affects that of $W(y)$ itself depends on the form of $U(y)$ (see expressions (7) and (8) below), so the concavity of $U(y)$ itself can be described as having subtle effects. The statement in the text and others like it, however, are offered in the hope of communicating the underlying intuition, which can be understood as taking the utilitarian case (no concavity of $W(U)$ ) as a benchmark and asking how increasing the concavity of $W(U)$ relative to this zero baseline affects the overall social aversion to inequality in the distribution of income. In any event, the central claim that the concavity of utility and the concavity of welfare have asymmetric effects stands.
} 
income redistribution. This does not, however, imply that the concavity of $U(y)$ is similarly inconsequential, for if that function is reasonably concave, there could still be a substantial difference between the marginal utility of income of different individuals, for it is not assumed that there are no significant differences in income. Put another way, the identify function for income - which is what $U$ is a function of - has a slope of one whereas the function $U(y)$ - which is what $W$ is a function of - has a slope near zero in this hypothetical setting. Marginal utilities may differ greatly even when utility levels do not.

For example, in the case in which $U(y)=\ln y$, and $y$ is $\$ 10,000$ for a poor person and $\$ 100,000$ for a high-income person, the ratio of marginal utilities (poor to rich) is 10 to 1 (marginal utility equals the inverse of income). By contrast, the resulting ratio of utility levels is only 1.25 to 1 . If the function $W(U(y))=\ln U(y)$ - that is, if the concavity of the welfare function is the same as the concavity of the utility function - the concavity of the welfare function will not add nearly as much to the aggregate social value of income redistribution as was already present on account of the concavity of the utility function. In this example, the ratio of $W^{\prime}(10,000)$ to $W^{\prime}(100,000)$, the relevant derivatives of $W(y)$ with respect to $y$, is 12.5 to 1 , so the overall value of income redistribution is $25 \%$ greater than if the welfare function were linear.

The result in the foregoing example extends to varying degrees of concavity of utility and welfare functions. Moreover, it does not depend on the assumption that the utility or welfare functions are of the constant-elasticity form. However, the result is not general in another, quite important sense, for it depends on certain aspects of the parameterization of the functions, as will be discussed. It turns out that the ultimate resolution involves empirical questions pertaining to individuals' utility functions.

For completeness and concreteness, the analysis will be applied to standard (constantelasticity) forms of utility and welfare functions in section 3. It will be shown that there are some complications that arise regarding the welfare function. Overcoming them is not technically difficult, although the resulting form of the welfare function deviates from the norm in important and possibly problematic ways. In section 4 , the functional forms and parameterizations used in some of the most-cited optimal income taxation simulations will be examined in light of the present analysis. It will be seen that the results attributed to intermediate degrees of social aversion to inequality in the distribution of income can better be rationalized by reference to the concavity of individuals' utility functions than with regard to the concavity of welfare as a function of utilities. Concluding remarks address the need for empirical work on certain features of individuals' utility functions and comment on debates about the appropriate form of the social welfare function.

\section{Analysis}

2.1. Derivation. - It is useful to begin with some definitions. First, as it standard, the coefficient of relative risk aversion of $U(y)$ is 
(1) $C R R A=-\frac{y U^{\prime \prime}(y)}{U^{\prime}(y)}$.

Single and double primes denote first and second derivatives with respect to a function's argument. Second, let us analogously define the coefficient of relative utility-inequality aversion of $W(U)$ to be

(2) $C R U I A=-\frac{U W^{\prime \prime}(U)}{W^{\prime}(U)}$.

Importantly, the derivatives of $W$ here are with respect to $U$ (and not with respect to $y$ ) because we are referring to the concavity of the function $W(U)$ in terms of $U$. Just as CRRA is a measure of the concavity of $U$ as a function of $y$, CRUIA is a measure of the concavity of $W$ as a function of $U$. (As noted, if CRUIA $=0, W(U)$ is not strictly concave; it is linear, that is, utilitarian.) Third and finally, define the coefficient of relative income-inequality aversion of the composite function $W(U(y))$, usually denoted herein simply as $W(y)$, to be

(3)

$$
\operatorname{CRIIA}=-\frac{y W^{\prime \prime}(y)}{W^{\prime}(y)}
$$

Here (by contrast to expression (2)), since we are referring to $W(y)$, the derivatives of $W$ are with respect to $y$. This expression indicates the concavity of the composite function $W(y)$ with respect to its argument, income. This is an indication of the overall social preference for income redistribution.

The first measure, CRRA, is a feature of individuals' utility functions that in principle is observable; the second measure, CRUIA, reflects a social value judgment; and CRIIA is a combination of the two. The task here is to determine precisely what that combination is and to interpret the result. To begin, the derivatives of the composite function $W(U(y))$ are

(4) $\frac{d W(U(y))}{d y}=W^{\prime}(U) U^{\prime}(y)$, and

(5) $\frac{d^{2} W(U(y))}{d y^{2}}=W^{\prime}(U) U^{\prime \prime}(y)+W^{\prime \prime}(U) U^{\prime}(y)^{2}$.

Therefore, 
(6)

$$
\begin{aligned}
C R I A & =-\frac{y\left(W^{\prime}(U) U^{\prime \prime}(y)+W^{\prime \prime}(U) U^{\prime}(y)^{2}\right)}{W^{\prime}(U) U^{\prime}(y)} \\
& =-\frac{y U^{\prime \prime}(y)}{U^{\prime}(y)}-\frac{y U^{\prime}(y) W^{\prime \prime}(U)}{W^{\prime}(U)}
\end{aligned}
$$

The first term of the latter expression is simply CRRA. The second term is similar to CRUIA. Multiplying it by $U(y) / U(y)$ and substituting yields

(7) $C R I A=C R R A+\left(\frac{y U^{\prime}(y)}{U(y)}\right) C R U I A$.

For subsequent discussion, it is useful to note that the first factor of the second term is equivalent to the ratio of the marginal utility of income, $U^{\prime}(y)$, to the average utility of income, $U(y) / y$. It is also the elasticity of utility with respect to income $\eta$, which allows us to rewrite expression (7) as

$$
C R I I A=C R R A+\eta C R U I A
$$

Thus, using relative-risk-aversion-like measures of concavity, the overall concavity is not simply the sum of the concavity measures of the utility and welfare functions; instead, the latter is weighted by the elasticity of utility with respect to income. ${ }^{7}$

2.2. Interpretation. - Expressions (7) and (8) are in accord with the intuition and example presented in the introduction. The overall social preference for income redistribution the degree of aversion to income inequality - is a combination of the concavity of utility individuals' aversion to risk or, equivalently, the rate at which marginal utility falls with income - and the concavity of welfare - society's aversion to inequality in the distribution of utilities but the latter is not simply added. Instead it is weighted by $\eta$, equivalently, the ratio of marginal to average utility. Now, it might be supposed - and was true in the example - that this ratio falls as income rises and, if utility is reasonably concave, becomes very small when incomes are high.

Consider again the illustration in which $U(y)=\ln y$ and $W(U)=\ln U$. In this case,

\footnotetext{
${ }^{7}$ If one wished instead to use absolute-risk-aversion-like measures of curvature, as is done in some of the literature, one could use expressions (4) and (5) to form an analogue to expression (6) for the coefficient of absolute income-inequality aversion. Then, making appropriate substitutions yields

CAIIA= CARA+U' CAUIA,

where the acronyms have analogous meanings to those in the text. As will become clear in the discussion to follow, the interpretation would be similar. Here, as income rises, $U^{\prime}$ becomes small, so CAUIA (the curvature of the welfare function) becomes relatively unimportant. (However, since $U^{\prime}$ is always positive, there is no complication corresponding to the possibility that $\eta$ may be negative below a certain level of income.)
} 
CRRA $=1$, CRUIA $=1$, and $\eta=1 /(\ln y)$. Thus, CRIIA $=1+1 /(\ln y)$. When $y$ is large, $\eta$ is small, indeed, approaching zero in the limit, in which case CRIIA approximately equals CRRA (unless CRUIA is truly extreme, such as in the case of maximin, in which case CRUIA is infinite). For the values of $y$ in the example, 10,000 and 100,000, the values of $\ln y$ are approximately 9.2 and 11.5 , and the values of $\eta$ are approximately 0.1 in either case. Thus, given the concavity of the utility function, the concavity of the welfare function adds little in determining the overall social aversion to income inequality.

In fact, most estimates of CRRA in various empirical literatures are at least 1.0 (corresponding to the concavity of the $\ln y$ utility function), many economists believe that CRRA is likely in the range of 1.0 to 2.0, and numerous empirical estimates (many from finance literature on the equity premium) suggest that CRRA's may be far higher than this. ${ }^{8}$ In any event, it would appear that plausible values for CRRA indicate a good deal of concavity of the utility function, making the present discussion applicable. If this were the entire story, it would seem therefore that differences in views concerning the appropriate welfare function - notably, whether one should follow Harsanyi $(1953,1955)$ and others in accepting a utilitarian social welfare function or instead should believe in a good degree of additional concavity, perhaps a CRUIA of one or two - may not make much difference. As long as $\eta$ is fairly small, the overall social preference for redistribution, reflected in CRIIA, would not be greatly affected.

Another implication of expressions (7) and (8) is that the relative importance of concavity in the welfare function, CRUIA, will depend on income level. Of course, it is obvious that income levels may be relevant because CRRA and CRUIA need not be constant, as in many standard analyses, but could vary with the level of income and utility, respectively. The point here is that there is an additional channel of influence, through $\eta$. Indeed, in the present example, both CRRA and CRUIA are constant (equal to one), but $\eta$ is falling with income. This suggests that the relative importance of concavity of welfare may be greater at lower income levels. Thus, positing a welfare function somewhat more averse to inequality in utility levels may be more important concerning, say, redistribution between lower-income individuals and the very poor (which is particularly relevant, for example, to the level of income guarantees and phaseout rates) than it is for, say, how much of the tax burden should be borne by the middle class versus the rich (which is central to debates about rate graduation at middle- and upperincome levels).

The interpretation of expressions (7) and (8), however, is not nearly as straightforward as the foregoing implies. The reason is that the suggestion that $y$ is "high" is obscure, indeed arguably meaningless, because utility functions like ln $y$ require specification of the units in which y is measured. If $y$ were measured not in dollars but in thousands of dollars, the values of $\ln y$ would be approximately 2.3 and 4.5 (instead of 9.2 and 11.5), and the values of $\eta$ would be approximately 0.4 and 0.2 (instead of 0.1 ); going further, if $y$ were measured in ten thousands, the individual with income of 10,000 would have a utility of 0 and $\eta$ would be infinite. Even higher units would yield negative values for utility and $\eta$.

\footnotetext{
${ }^{8}$ See, for example, the literature cited in note 4.
} 
This is not a mere mathematical anomaly but instead reflects a relevant aspect of reality. Recall the initial suggestion, using expression (7), that $\eta$ is plausibly low because marginal utility tends to be much less than average utility. When income is sufficiently low, this may not be so. Thus, if we think of a subsistence level of income, or perhaps of a level below which life would be so miserable that it would not be worth living, we would have utility of zero and thus average utility of zero; the ratio of marginal to average utility would indeed be infinite. (And at lower income levels, the ratio would be negative.) Just above this level, the ratio of marginal utility to average utility would be quite high. This ratio would fall - at first rapidly then more slowly, for standard functional forms - as income rises, eventually approaching zero.

If the choice of units of measurement were merely a matter of the analysts' discretion, the situation would be highly problematic. However, the question of the relevant units and functional forms is ultimately an empirical one. For example, individuals' behavior that affects their risk of death implicitly reveals information about utility levels (relative to a zero point). ${ }^{9}$ Thus, if one could determine a functional form (perhaps from other behavior), one could combine further evidence to make the necessary calibrations. Only then could one determine whether income was "low" or "high," and therefore the magnitude of $\eta$ and thus how relatively important or unimportant was the concavity of welfare in augmenting the concavity of utility in determining the overall degree of social aversion to income inequality.

\section{Application: Constant-Elasticity Functional Forms}

This section considers the relationship between constant-elasticity forms for the utility function $U(y)$ and welfare function $W(U)$ on one hand and for the reduced-form welfare function $W(y)$ on the other hand. In particular, it asks whether the constant-elasticity reduced-form welfare function is simply a composite of underlying functions of the same form, what modifications may be necessary if it is not, and how the results may be interpreted in light of the general question under consideration.

\subsection{Derivation. - Suppose that $U(y)$ and $W(U)$ each take the standard constant-elasticity} form.

(9) $U(y)=\frac{y^{1-x}}{1-\alpha}$,

where $\alpha$ is the coefficient of (constant) relative risk aversion.

(10) $W(U)=\frac{U^{1-e}}{1-e}$,

where $e$ is the coefficient of (constant) relative utility-inequality aversion. In this case, the

\footnotetext{
${ }^{9}$ See, for example, Kaplow (2003).
} 
composite function, $W(U(y))$, or $W(y)$, is

$$
\text { (11) } \begin{aligned}
W(y) & =(1-\alpha)^{e} \frac{y^{(1-\alpha)(1-e)}}{(1-\alpha)(1-e)} \\
& =(1-\alpha)^{e} \frac{y^{1-\gamma}}{1-\gamma},
\end{aligned}
$$

where $\gamma$ is the coefficient of (constant) relative income-inequality aversion, which here equals $1-(1-\alpha)(1-e)$. Note that, from (8), we also know that $\gamma$ must equal $\alpha+(1-\alpha) e$, using the fact that when $\alpha$ is the (constant) CRRA, $\eta=1-\alpha$. These expressions for $\gamma$ are indeed equivalent.

Three observations should be noted. First, expression (10) for $W(U)$ is not in general well defined. Specifically, we are interested in cases in which $\alpha$ may exceed one (as is empirically plausible). From expression (9), this means that $U(y)$ is negative; in (10), $U$ is raised to a real exponent, which is an undefined operation (unless the exponent is an integer). Second, in (11), the same problem arises because $1-\alpha$ is also raised to the real exponent $e$. Third, consider the latter expression for $\gamma$, that is $\gamma=\alpha+(1-\alpha) e$. For the case under discussion $(\alpha>1)$, a higher value of $e$ reduces rather than increases the overall curvature of $W(y)$, reflected in $\gamma$. (Similarly, when $e>1$, as $\alpha$ rises, $\gamma$ is falling rather than increasing.)

In sum, combining a standard constant-elasticity functional form for $U(y)$ and $W(U)$ does not yield a simple constant-elasticity reduced-form expression for $W(y)$ that has the characteristic that one can combine the curvature measures for the two functions, $\alpha$ and $e$, in the expected manner to yield the curvature for the reduced-form expression. To address this, supposes instead that we work backwards, asking what form $W(U)$ must take in order to get the standard constantelasticity expression for the reduced-form

$$
W(y)=\frac{y^{1-\gamma}}{1-\gamma}
$$

Sticking with the constant-elasticity utility function in expression (9), the required form for the welfare function is

$$
\text { (13) } \begin{aligned}
W(U) & =(1-\alpha)^{-e} \frac{U^{1-e}}{1-e} \\
& =\frac{[(1-\alpha) U]^{-e} U}{1-e} .
\end{aligned}
$$


The former (upper) expression in (13) is more natural, and when compared with expressions (11) and (12), it is clear that expression (13) will produce the desired result. The latter (lower) rearrangement in (13) is offered because it more clearly addresses the question whether a negative base is being raised to a real exponent. Recall from expression (9) that when $\alpha>1$, so that $1-\alpha<0$, it will be true that $U<0$, so the term in square brackets will still be positive. ${ }^{10}$ Finally, it is straightforward to verify that expression (13) for $W(U)$ is indeed of constantelasticity form, with a CRUIA of $e$ as before.

3.2. Interpretation. - Although a rationalization, of sorts, for the standard constantelasticity reduced-form for $W(y)$, as represented in expression (12), has been offered, it nevertheless deviates from what one may have expected in two important respects.

First, expression (13) for $W(U)$ is not formally an individualistic social welfare function. The reason is that an individualistic social welfare function is in principle a mapping, from the space of individuals' utility levels to the real line, that does not depend on how those utility levels were generated. In expression (13), however, in addition to the utility level one must also know a parameter of the individual's utility function, $\alpha .{ }^{11}$ That is, the expression here for $W(U)$ will not generate the same measure independently of how the individual's utility arises. (For example, if two individuals had the same utility level, one highly risk averse and subject to modest risk and the other less risk averse but subject to a greater risk, the social welfare ascribed to each would differ under the present formulation.) This deviation from individualism might be deemed objectionable per se or simply lacking in affirmative justification. (Why should a more risk-averse individual's utility count, say, less than others' utility in assessing social welfare?) Moreover, one might find problematic the fact that such a non-individualistic social welfare function violates the Pareto principle. ${ }^{12}$

Second, the curvature of the reduced-form welfare function $W(y)$ does not superficially appear to have the desired property, namely, that one in a sense adds the curvatures of the utility function $U(y)$ and of the welfare function $W(U)$. Recall that the concavity parameter (CRIIA) for $W(y)$ is $\gamma=\alpha+(1-\alpha) e$. Again considering the case in which $\alpha>1$, it is clear that, the higher is $e$, the lower is the composite curvature parameter $\gamma$. For example, if $\alpha$ and $e$ each equal $2, \gamma$ equals zero (not some number larger than 2 , such as 4 ).

This latter difficulty, however, is more illusion than reality. The reason relates to the fact that the standard-form constant-elasticity utility function in expression (9) is, as previously remarked, negative in this case. If one considers negative values for $e$ and uses them in

\footnotetext{
${ }^{10}$ As is familiar, when $\alpha=1$, it is conventional to take the expression for utility to be $\ln y$, which does have a constant CRRA of 1 . (When -1 is added to the numerator in (9), the limit of $U(y)$ as $\alpha$ approaches 1 , using l'Hôpital's rule, is $\ln y$.)

${ }^{11}$ Although it is permissible to scale individuals' utility functions to provide interpersonal comparability, only linear transformations are allowed on that account. The coefficient $\alpha$ concerns the curvature of individuals' utility functions, which as noted earlier, pertains to observable behavior.

${ }^{12}$ See Kaplow and Shavell (2001). They show that continuity of the welfare function in utility levels is a sufficient condition for a violation of the Pareto principle, and of course the welfare functions under consideration here are continuous.
} 
expression (13), and if one further confines attention to the actual domain of the function (negative real values), the properties of this $W(U)$ are as desired. Consider, for example, the case in which $\alpha=2$ and $e=-2$. Then $\gamma=4$. Furthermore, examining the upper expression in (13), one can see that the lead coefficient will equal 1 and the other factor will be $U^{3} / 3$. Recalling that $U$ is negative, we are in a concave and upward sloping region for this function. Likewise, one could consider the same case except that $e=-1$; then $\gamma=3$, the lead coefficient in the upper expression in (13) is -1 , the other factor will be $U^{2} / 2$, and because $U$ is negative we are again in a concave and upward-sloping region for this function.

The present complications arise because the standard constant-elasticity form for the utility function, expression (9), is negative when $\alpha>1$. One could have shifted the function up (without affecting the constant CRRA of $\alpha$ ) by simply adding a constant. More precisely, consider

$$
U(y)=\frac{y^{1-\alpha}-z}{1-\alpha}
$$

(Although $z$ is subtracted in the numerator, it is divided by $1-\alpha$, which is negative in the cases with which we are presently concerned.) In this case, however, the function no longer has constant elasticity. Instead, one can show that

$$
\eta=(\alpha-1) \frac{1}{z y^{\alpha-1}-1}
$$

In this case, $\eta$ will be positive (rather than negative, as above) as long as $z y^{\alpha-1}>1$, which will be true for a given (positive) $z$ as long as $y$ is sufficiently large. Accordingly, $e$ will add to rather than subtract from $\gamma$, the concavity of the reduced-form welfare function, $W(y)$. Furthermore, as $y$ becomes larger, the impact of $e$ will become increasingly unimportant (zero in the limit).

The foregoing is reminiscent of the discussion in section 2 , where the possibility that utility might be negative below a certain level of $y$ was raised and the suggestion was made that $\eta$ might plausibly be falling with income. Recall, however, that the ultimate choice of parameters and functional form for $U(y)$ in the present setting (including the units in which $y$ is to be measured) is not a matter of the analyst's convenience but rather needs to be determined empirically.

\section{Application: Optimal Income Taxation Literature}

The preceding section raises questions about whether conventional constant-elasticity reduced forms for the social welfare function, $W(y)$, can be rationalized in terms of similar functional forms for the underlying utility and welfare functions. In addition, sections 2 and 3 both suggest that it may be problematic to interpret a concavity parameter for such a reducedform function as a simple combination of the concavities of the utility and welfare functions. This section applies the foregoing analysis to the functional forms employed in the literature that 
presents numerical simulations for optimal income taxation.

Mirrlees (1971) functional form for individuals' utility is

$$
U(c, l)=a \ln c+\ln (1-l)
$$

where $c$ is consumption and $l$ is labor supply. He sets the constant $a$ equal to 1 in his simulations. Individuals' marginal utility of consumption is $1 / c$ in this case, and this utility function has a constant CRRA of 1 . Mirrlees uses a constant absolute utility-inequality aversion form for the welfare function,

(17) $G(U)=-\frac{1}{\beta} e^{-\not U U}$,

where $\beta$ is the absolute utility-inequality aversion parameter. For $\beta=0$, he defines $G(U)=U$.

Mirrlees presents simulations for $\beta$ 's of 0 and 1 . One can show that the overall curvature (CRIIA, corresponding to $\gamma$ ) for this case is $1+\beta .^{13}$ Hence, what might be viewed as his utilitarian case $(\beta=0)$ has significant social aversion to inequality. (Concretely, an individual with ten times the consumption of another has marginal utility and thus a marginal social valuation a tenth as high as that of the other individual.) Interestingly, in subsequent writing, Mirrlees (1982, p. 77 n. 21) clearly states that, as he endorses a utilitarian welfare function, he intended $G(U)$, expression (17), to be taken as a utility function, suggesting that $\beta$ is an observable preference parameter, not a social judgment. From that perspective, the difficulty addressed herein concerning the effects of concavity of utility and of welfare does not arise.

Atkinson (1973), another of the most cited articles on the subject, employs an approach formally close to that of Mirrlees (1971). The main difference is that Atkinson in essence considers a constant-elasticity reduced-form welfare function, as in section 3 above, and specifically considers whether a high value of $e$ may make optimal significantly higher tax rates than Mirrlees found possible to justify. (His answer is affirmative.)

More precisely, in his simulations Atkinson uses a utilitarian social welfare function with utility an iso-elastic function of income. He then presents the most significant interpretative discussion in the literature. ${ }^{14}$ He begins by observing that it is unclear whether the curvature

\footnotetext{
${ }^{13}$ More generally, if the utility function is of the form in expression (16) except that the first term exhibits constant relative risk aversion of $\alpha$ (which need not equal 1), the overall curvature for this case is $\alpha+\mathrm{c}^{1-\alpha} \beta$. As with the functional forms explored in section 3 , for the case of $\alpha>1$, curvature of the welfare function becomes relatively unimportant as $c$ becomes large (again raising the question of the units in which $c$ is measured). Note also that with the functional form for the welfare function in expression (17), $U$ is the exponent rather than the base, as in expression (10), so the possibility that $U$ is negative raises no technical problems.

${ }^{14}$ See also the discussion in Atkinson (1970. p. 257) on the use of social welfare functions in the normative measurement of inequality.
} 
parameter can be estimated from individuals' behavior. (Whether he has in mind conceptual or practical limitations is not stated.) He proceeds to introduce and develop the idea that the parameter "may reflect social values as well as individual utility." (Atkinson 1973, p. 104.) Following this, he discusses literatures that may provide a basis for attaching a social value to aversion to inequality. Atkinson's discourse is perhaps most responsible for the current, now conventional view that one may interpret curvature parameters in a reduced-form social welfare function as reflecting some combination of curvature of the implicitly underlying utility and welfare functions. He does not, however, derive a composite social welfare function that incorporates this view, which section 3 indicates is not an entirely straightforward exercise given the desired interpretation.

Perhaps the most-cited simulation results are those of Stern (1976), who calculates optimal linear income tax schedules as a function of various parameters, including one indicating how the social marginal valuation of income changes with income. ${ }^{15}$ Specifically, the utility function he employs is

$$
U(c, l)=\left(a(1-l)^{-\mu}+(1-a) c^{-\mu}\right)^{-1 / \mu}
$$

This function is of CES form, with an elasticity of substitution of $1 /(1+\mu)$. This utility function is homogenous of degree one (linear) in leisure (1-l) and consumption $(c)$. The social welfare function is

(19) $S=\frac{1}{v} \int_{0}^{\infty} U^{v}\left(c_{n}, l_{n}\right) f(n) d n$,

where $v$ corresponds to 1-e in expression (10) for $W(U), f$ is the density function, and $n$ indicates an individual's type. Stern uses different choices of $v$ to correspond to different social judgments about the importance of inequality. Specifically, he examines values of 1 (utilitarian), $-1,-2$, and $-\infty$ (maximin).

One can best understand the role of concavity in utility and welfare in Stern's analysis by considering the results for the $v=1$ (utilitarian) case, in which there is "no special preference for equality [and accordingly] the redistributive benefits are very small" (Stern 1976, p. 153). Of course, it hardly follows a priori that in the utilitarian case redistributive benefits are small. (See, for example, Mirrlees (1971), discussed above.) How great they are depends on individuals' CRRA, or $\alpha$ in the case in which it is constant. Stern's utility function, however, is quasi-linear. Because it is homogenous of degree one, scaling up leisure and consumption in proportion increases utility in the same proportion. This function is not actually linear in consumption, however, because leisure does not scale up in proportion. Instead, leisure acts (very roughly) as a

\footnotetext{
${ }^{15}$ Stern's results are reported, for example, in Atkinson and Stiglitz (1980), Myles (1995), and Tuomala (1990), and his functional forms are used in other simulations, such as that by Slemrod et al. (1984) for the optimal two-bracket income tax.
} 
constant. Hence, increasing $c$ increases $U$ less than proportionately, introducing some concavity. Nevertheless, given his parameterizations ( $c$ is very low, on the order of 0.1 to 0.2 in some simulations, even lower in others), this effect is not very large. Thus, the small redistributive benefit in Stern's simulations for the utilitarian case reflects that individuals' utility functions are stipulated to be only moderately nonlinear in consumption.

Suppose, however, that individuals' marginal utility of income diminishes a good deal more rapidly than this, such as in the case of a constant CRRA of 2 . In that instance, one might take what is deemed to be the social welfare function, expression (19), with $v=-1$, as the individual's utility function. If one does so, the CRRA for the resulting utility function indeed appears to have a value near 2. ${ }^{16}$ This result can also be understood from expression (8), stating that CRIIA $=$ CRRA $+\eta$ CRUIA. Taking the limiting case in which $U$ is linear, CRRA $=0$ and, for this sort of parameterization, $\eta=1$, so CRIIA $=$ CRUIA. And when CRRA is small rather than $0, \eta$ will be somewhat less than 1 , and it will then be true that, within a range, CRIIA $\approx$ CRUIA.

It is more difficult, however, to interpret Stern's formulation if neither CRRA nor CRUIA is near zero. That is, although one might well choose some $v$ in Stern's formulation to correspond to individuals' CRRA, the association of higher values of $v$ with more concave versions of a welfare function like Stern's (expression 19) or that in section 3 (expression 10) is problematic, as we have seen. Also troubling are comments like: "I prefer $v=-1$, corresponding to an assertion that the social marginal valuation of income should decrease as the square of income." (Stern 1976, pp. 151-152.) In addition to the aforementioned worries, such an assertion, in his reduced-form specification of the social welfare function, mixes empirical judgments about individuals' utility functions and normative judgments about the welfare function. The former, of course, is not a matter of the analyst's preferences. The latter may be, but ultimately must be justified. Additionally, such statements implicitly suggest that the analyst's normative principle might change depending on the facts. For example, if individuals in fact were nearly risk-neutral, the stated preference corresponds to a view that utilitarianism is insufficiently egalitarian, but if individuals were discovered instead to have CRRA's in excess of 2 , as some evidence suggests, the stated preference implies that utilitarianism is too egalitarian.

\section{Conclusion}

The overall marginal social value of redistributing income depends on both the concavity of individuals' utility functions and the concavity of the social welfare function. The foregoing analysis explores the precise relationship between the concavity of these two underlying functions and the concavity of the composite, reduced-form expression for social welfare as a function of income that appears in much of the pertinent literature. In considering both the general case, standard constant-elasticity functional forms, and the specific versions used in the

\footnotetext{
${ }^{16}$ Stern does not report all the parameter values necessary to compute this, but using central values for those reported and values that seem plausible in light of what is reported for other parameters, my calculations for two cases (one chosen to be on the high side and one low) were both very close to 2.0.
} 
literature presenting numerical simulations for determining optimal income tax rates, it is found that conventional interpretations based on the reduced form are not straightforward and can be misleading. Some results are more readily rationalized if the basis for social aversion to income inequality is rooted in the concavity of individuals' utility functions rather than in the concavity of the social welfare function.

The analysis makes clear that empirical work should have a greater role in calibrating overall social judgments concerning income inequality. Notably, the importance of a significantly utility-inequality averse social welfare function (short of extremes, approaching maximin) is not additive in a simple manner but instead depends importantly on aspects of individuals' utility functions. Specifically, it is necessary to ascertain the elasticity of utility with respect to disposable income (consumption). This in turn may depend greatly on whether individuals' incomes are sufficiently low to be near a point at which utility is close to zero in a meaningful absolute sense that is in principle ascertainable from behavior, such as that concerning how individuals value life-preserving precautions as a function of their income.

In addition, depending on what such empirical work reveals, it may be that the degree of utility-inequality aversion expressed in the social welfare function (again, short of extremes) is not very important relative to the curvature of individuals' utility functions. If so, empirical evidence on that curvature is particularly important in formulating operational social judgments about inequality. Likewise, debates about whether the proper social welfare function is utilitarian or strictly concave (and, if so, how concave) may have diminished practical significance. Considerations of concavity of the reduced-form social welfare function would to a substantial extent take a place alongside assessments of the elasticity of labor supply and of the distribution of skills as empirical determinants of the level and shape of optimal income tax schedules. 


\section{References}

ATKINSON, A. B. (1970), “On the Measurement of Inequality”, Journal of Economic Theory, II, 244-263.

ATKINSON, A. B. (1973), "How Progressive Should Income Tax Be?", in F. Parkin \& A.R. Nobay (eds.) Essays on Modern Economics, Chapter 6 (London: Longman) 90-109.

ATKINSON, A. B. and STIGLITZ, J. E. (1980) Lectures on Public Economics (New York: McGraw-Hill).

BARSKY, R. B., JUSTER, F. T., KIMBALL, M. S. and SHAPIRO, M. D. (1997), "Preference Parameters and Behavioral Heterogeneity: An Experimental Approach in the Health and Retirement Study", Quarterly Journal of Economics, CXII, 537-579.

BLACKORBY, C., BOSSERT, W., and DONALDSON, D. (1999), "Income Inequality Measurement: The Normative Approach," in J. Silber (ed.) Handbook of Income Inequality Measurement, Chapter 3 (Boston: Kluwer Academic Publishers) 133-157.

BOSKIN, M. J. and SHESHINSKI, E. (1978), “Optimal Redistributive Taxation when Individual Welfare Depends upon Relative Income”, Quarterly Journal of Economics, XCII, 589-601.

CAMPBELL, J. Y. (1996), “Understanding Risk and Return,” Journal of Political Economy, CIV, 298-345.

CHOI, E. K. and MENEZES, C. F. (1992), “Is Relative Risk Aversion Greater than One?”, International Review of Economics and Finance, I, 43-54.

HARSANYI, J. C. (1953), "Cardinal Utility in Welfare Economics and in the Theory of RiskTaking”, Journal of Political Economy, LXI, 434-435.

HARSANYI, J. C. (1955), "Cardinal Welfare, Individualistic Ethics, and Interpersonal Comparisons of Utility”, Journal of Political Economy, LXIII, 309-321.

HOCHMAN, H. M., and RODGERS, J.D. (1969), "Pareto Optimal Redistribution”, American Economic Review, LIX, 542-557.

KAPLOW, L. (2003), "The Value of a Statistical Life and the Coefficient of Relative Risk Aversion" (NBER Working Paper 9852).

KAPLOW, L. and SHAVELL, S. (2001), “Any Non-welfarist Method of Policy Assessment Violates the Pareto Principle", Journal of Political Economy, CIX, 281-286.

KOCHERLAKOTA, N. R. (1996), “The Equity Premium: It's Still a Puzzle”, Journal of Economic Literature, XXXIV, 42-71.

MIRRLEES, J. A. (1971), “An Exploration in the Theory of Optimal Income Taxation”, Review of Economic Studies, XXXVIII, 175-208.

MIRRLEES, J. A. (1982), “The Economic Uses of Utilitarianism”, in A. Sen and B. Williams (eds.) Utilitarianism and Beyond, Chapter 3 (Cambridge: Cambridge University Press) 63-84. 
MYLES, G.D. (1995) Public Economics (Cambridge: Cambridge University Press).

SLEMROD, J., YITZHAKI, S., MAYSHAR, J. and LUNDHOLM, M. (1994), "The Optimal Two-Bracket Linear Income Tax”, Journal of Public Economics, LIII, 269-290.

STERN, N.H. (1976), "On the Specification of Models of Optimum Income Taxation”, Journal of Public Economics, VI, 123-162

TUOMALA, M. (1990) Optimal Income Tax and Redistribution (Oxford: Oxford University Press). 